\title{
FEATURE REDUCTION OF SHORT TEXT CLASSIFICATION BY USING BAG OF WORDS AND WORD EMBEDDING
}

\author{
Narongsak Chayangkoon ${ }^{1}$, Anongnart Srivihok ${ }^{2}$ \\ Department of Computer Science, Faculty of Science, Kasetsart University, \\ Bangkok and Thailand \\ ${ }^{1}$ narongsak.chay@ku.th, ${ }^{2}$ fsciang@ku.ac.th
}

\begin{abstract}
Text classification for data preprocessing methods regularly uses bagof-words (BoWs). In a large dataset, BoWs always include many vectors with very large sizes and high dimensions. The authors introduced a new data preprocessing method for feature reduction of short text classification, namely NDTMD. It reduces features of the dataset using BoWs and word embedding (WE), and can solve the weaknesses of BoWs. The experiment consisted of four steps: 1) 5 datasets were selected from the data science community website, Kaggle; 2 ) the new methods were compared with 5 commonly used data preprocessing methods and 4 of these 5 methods used the state of the art as their baseline, while the other one used BoWs. One of the new data preprocessing methods used features reduction of BoWs to produce a new document termed matrix data (NDTMD); 3) the authors generated many classification models by 3 classifiers: support vector machine, logistic regression, and convolutional neural network for text classification; and 4) the above classifiers were applied to each preprocessing dataset and evaluated using feature reduction rate (FRR), accuracy, kappa, and running time performance. The results showed that classification models had the highest performance when using NDTMD. In particular, classifier algorithms had the highest accuracy and kappa but the lowest running time. The new data preprocessing methods can be used to preprocess short text classification and also can be applied with real social media data.
\end{abstract}

Keywords - feature reduction, word embedding, text classification, bag-of-words

\section{INTRODUCTION}

The bag-of-words (BoWs) representation is widely accepted technique in text classification tasks. This representation considers the frequency of each word as a feature in the classification of data and encoding every word as a one-hot representation. Each word is represented by a sparse vector comprised of its index and frequency [1]. Thus, as features may potentially run into many vectors, BoWs pose large scalability challenges and BoWs is a high dimensional vector [2].

To address this major problem in using BoWs, this article proposed a new data preprocessing method for the feature reduction of short text classification. The method is the features reduction of BoWs which was performed by using a statistical function to obtain the preprocessed dataset with smaller dimensions. Furthermore, this processing used intersected features of BoWs and words in word embedding (WE) which were generated from the corpus using the Word2vec software. This method produced a dataset

Received: December 15, 2018

Reviewed: February 5, 2019

Accepted: February 10, 2019 
called new document term matrix data (NDTMD). In the experiment, the authors compared the classification performance of NDTMD with various state of the art methods, consisting of: term frequency-inverse document frequency (TF-IDF) [3], latent semantic analysis (LSA) [4]-[5], paragraph vector-distributed memory model (PV-DM) [6], paragraph vector without word ordering-distributed bag of words (PV-DBOW) [6] and baseline.

The classifiers consisted of support vector machine (SVM), Logistic regression and convolutional neural network (CNN). The criterion classification used the accuracy, kappa, and running time performance. Furthermore, the feature reduction performances were evaluated using the feature reduction rate (FRR).

\section{RELATED WORK}

\subsection{BAG-OF-WORDS (BoWs)}

BoWs is a linguistic context that it was first named by Harris (1954). This technique is a classic simplifying data preprocessing method. This model is a representation of the bag of words which is known as a vector space model. This model is the representation which is used for documentation of the occurrence of each word is used as a feature for training the classifier. This method encodes every word in one-hot representation. Each word represents a dimensionally sparse vector that has 1 at the corresponding index and the word is 0 at every other index [1].

\subsection{STATE OF THE ART METHODS}

\subsubsection{TERM FREQUENCY-INVERSE DOCUMENT FREQUENCY (TF-IDF):} TF-IDF is a data preprocessing method which uses the weight of words where this is intended to reflect the importance of words. This weight is a numerical statistical measure. It is also used to evaluate the importance of words in the archives. The term frequency (TF) and inverse document frequency (IDF) produce a composite weight for each word in each document (TF-IDF). The TF-IDF determines the weight of term (t) in the document (d). TF-IDF is represented by Equation (1) [3].

$\mathrm{TF}-\mathrm{IDF}_{t, d}=\left(\mathrm{TF}_{t, d} \times \mathrm{IDF}_{t}\right)$

2.2.2. LATENT SEMANTIC ANALYSIS (LSA): LSA is a theory and method in natural language processing (NLP) which is used for data preprocessing. This method extracts the relationships between documents and terms in the corpus by defining conceptual topics from groups of related terms. It assumes words that are closely related will occur in similar pieces of document. The calculation of the method used a mathematical technique called singular value decomposition (SVD). SVD is used to reduce the number of features while maintaining a similar meaning of each document. Thus, SVD is represented in Equation (2) [4]-[5].

$X=U \sum V^{T}$

Equation (2) is linear so the dissolution of $\mathrm{X}$. $\mathrm{U}$ and $\mathrm{V}$ are orthogonal matrices. Furthermore, $\sum$ is a diagonal matrix called SVD [4]-[5].

2.2.3. PARAGRAPH VECTOR-DISTRIBUTED MEMORY MODEL (PV-DM): PV-DM inserts the paragraph id as another word in an ordered sequence of words. PV-DM was first used by Le and Mikolov (2014). This model attempts to predict a word in the ordered sequence based on the other surrounding context words in the 
sentence and also the context provides by the paragraph id. The input word vectors are averaged or concatenated as part of the classification process [6]. The framework of model is shown in Fig. 1.

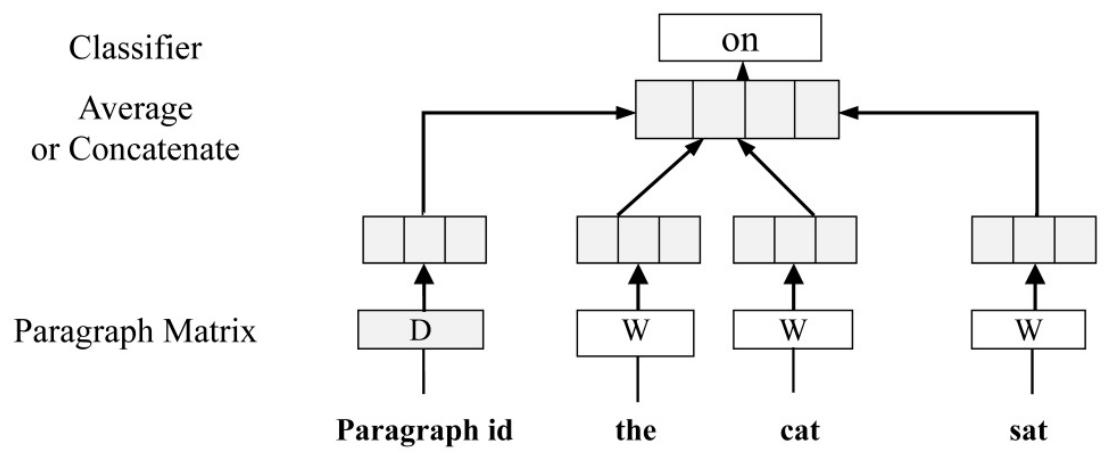

Fig. 1 Distributed memory model of paragraph vectors (PV-DM) [6]

\subsubsection{PARAGRAPH VECTOR WITHOUT WORD ORDERING -}

DISTRIBUTED BAG OF WORDS (PV-DBOW): PV-DBOW approaches the problem from the opposite direction to that used by Le and Mikolov (2014). PVDBOW takes a given paragraph id which is used to predict words in the window without any restriction on word order. Moreover, this version is trained to predict the words in a small window [6]. The framework of model is shown in Fig. 2.

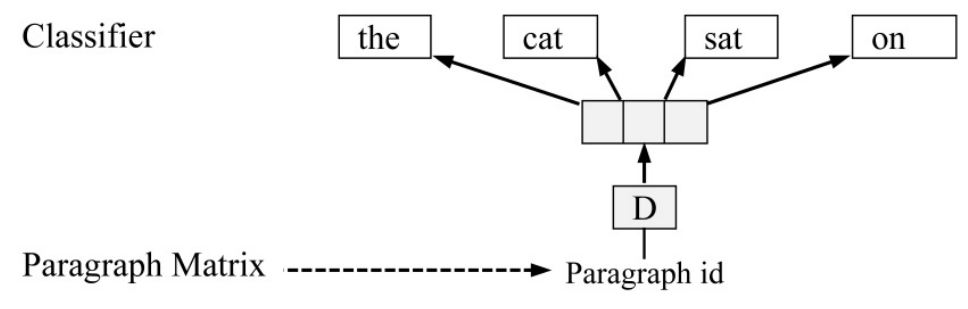

Fig. 2 Distributed bag of words version of paragraph vector (PV-DBOW) [6]

\subsection{WORD EMBEDDING}

Word embedding (WE) is the name for a set of methods where words are represented by a number vector. WE converts the words into numerical vectors. There are different numerical representations of words which depend on the context in which the word appears in the sentence [7]-[8].

\subsection{WORD2VEC}

Word2vec is software used to produce WE, it was developed by Mikolov et al. (2014). This software uses NLP that are trained to recreate the linguistic meaning of words. Word2vec uses a set of documents as its input. Then, this software produces vectors and several dimensions. Each distinct word in the corpus has a corresponding vector. Vectors are positioned by their context similarity according to the corpus [7]-[8].

Moreover, Word2 vec uses one of two architectures to create word distribution and representation. The first is the continuous bag-of-words (CBOW) model and the second is the continuous skip-gram model. Furthermore, according to Mikolov's suggestion, CBOW uses less time than skip-gram which is slower but does a better 
job for infrequent words. Moreover, the skip-gram model uses the current word to identify the window size of context words [9].

\subsection{SKIP-GRAM MODEL}

This model is known as a deep learning classification model which is a modified version of neural language model focused on training efficiency of WE. The skipgram model takes in the target word as input and tries to predict the context words. Then, the skip-gram model is trained to predict the surrounding words given the current word [7]-[8].

Jin et al. (2016) explained that the skip-gram model has two sets of WE which are defined for each word (w). The definition $\vec{v}(w)$ and $\vec{u}\left(w^{\prime}\right)$ is shown in Equation (3) to (5) [10].

$$
P(s)=\prod_{i=1}^{|s|} p\left(\vec{v}\left(w_{i}\right) \mid \vec{u}\left(w^{\prime}\right)\right)
$$

$\vec{u}(w)$ is context (input) of WE.

$\vec{v}(w)$ is target (output) of embedding vector of $w$. The target word is $w$, the context is $w^{\prime}$ and the probability of $\vec{v}(w)$ is given by $\vec{u}\left(w^{\prime}\right)$.

The model approximates a language model by approximating the probability of sentence. This definition is shown in Equation (4).

$$
\begin{gathered}
P(s)=\prod_{i=1}^{|s|} p\left(\vec{v}\left(w_{i}\right) \mid t\left(w_{i}, s\right)\right) \\
s=w_{1} w_{2} \ldots w_{|s|} \\
t\left(w_{\mathrm{i}}, s\right) \text { is the context of } w_{\mathrm{i}} \text { in } s .
\end{gathered}
$$

The skip-gram model is used to train WE efficiently and this model is shown in Equation (5).

$$
\begin{aligned}
P\left(\vec{v}\left(w_{i}\right) \mid t\left(w_{i}, s\right)\right) & =\prod_{w^{\prime} \in t\left(w_{i}, s\right)} P\left(\vec{v}\left(w_{i}\right) \mid \vec{u}\left(w^{\prime}\right)\right) \\
& =\prod_{w^{\prime} \in t\left(w_{i}, s\right)} \frac{e^{\vec{v}\left(w_{i}\right) \cdot \vec{u}\left(w^{\prime}\right)}}{\sum_{w^{\prime \prime} \in V} e^{\vec{v}\left(w^{\prime \prime}\right) \cdot \vec{u}\left(w^{\prime}\right)}}
\end{aligned}
$$

$\mathrm{P}\left(\vec{v}\left(w_{i}\right) \mid t\left(w_{i}, s\right)\right)$ is estimated by predicting $\vec{v}\left(w_{i}\right)$ using each word $w^{\prime}$ in $t\left(w_{i}, s\right)$. However, this approximation does not give a high accuracy.

The optimization of the skip-gram model is used to train WE efficiently. The result of WE can be used as training set for more sophisticated neural language models [7]-[8]. This optimization training is done by maximizing the likelihood of raw text using stochastic gradient descent. This is equivalent to maximizing $\nabla \log P\left(\vec{v}\left(w_{i}\right) \mid \vec{u}\left(w^{\prime}\right)\right)$ for all $w_{i}$ and $w^{\prime}$ in a corpus [8]-[10].

The gradients to the probability in Equation (3) require vocabulary and crossreferencing through a close estimation of the noise contrastive estimation (NCE) method which is shown in Equation (6) [10]-[11].

$\log \sigma\left(\vec{v}\left(w_{i}\right) \cdot \vec{u}\left(w^{\prime}\right)\right)+\sum_{j=1}^{l} E_{w_{j} \sim P_{n}(w)}\left[\log \sigma\left(-\vec{v}\left(w_{i}\right) \cdot \vec{u}\left(w^{\prime}\right)\right)\right]$

\subsection{WORD2VEC FOR TEXT CLASSIFICATION}

Lilleberg et al. (2015) proposed a method for improving the effectiveness of classification using Word2vec. Their method combined two preprocessing 
techniques: TF-IDF and Word2vec. In this study, TF-IDF technique was applied for Word2 vec dataset weighted by TF-IDF. Results showed the combination methods could outperform either individual method [12].

Kenter and Rijki (2015) published an article using word embedding which generated from short text unlabeled data. This method used semantic matching word level to text level based on semantic knowledge combined with word embedding. Terms were represented in a semantic space as the proximity of vectors and interpreted as semantic similarity. The results were evaluated using publicly available datasets commonly used in semantic similarity research. Their method was better than the baseline that tests under the same conditions [13].

\subsection{CLASSIFICATION ALGORITHMS}

2.7.1. SVM: SVM is a classification algorithm for two-class problems. The algorithm creates a decision boundary called a support vector as the divider for the two classes, making it a non-probabilistic binary classifier. Traditionally, SVM uses a linear function for assigning an instance vector to the class with least error according to the decision boundary. But a non-linear function can also be used by applying the kernel trick which transforms the inputs into a multi-dimensional feature space [14].

2.7.2. Logistic regression: Logistic regression is a statistical model of estimated coefficients for dichotomous variables. The model calculates the log-odd probability from a linear function of independent variables. Logistic regression can be used as a binary classifier by having the variable as one of the two possible classes [15].

2.7.3. CNN: $\mathrm{CNN}$ is a subclass of neural networks which have a high number of hidden layers. The hidden layers typically are 1) convolutional layers, which replicate the response of receptive fields for human eyes, 2) pooling layers, which combine the results of convolution layers, 3) fully connected layers, which associate every node from the previous layer to the next layer, and 4) the loss layer, which uses an activation function such as softmax to summarize the outputs from another layer into one value [16].

\section{EXPERIMENT}

The authors proposed a new data preprocessing method for the feature reduction of short text classification. The method was the features reduction of BoWs by the intersection between features of BoWs and words in WE and this new method produces a new dataset called NDTMD.

The experiment framework consisted of five steps. The first step selected 5 datasets from the data science community website, Kaggle; [17]. The second step was data preprocessing that involved three steps. Step 1, Preprocessing-Data (P1) was a corpus preparation. Step 2, Preprocessing Data (P2) involved various states of the art consisting of four methods: TF-IDF, LSA, PV-DM, and PV-DBOW. Step 3, Preprocessing Data (P3) used the new data preprocessing method (features reduction of the dataset using WE). First, BoWs was generated from the corpus, second WE was generated from the corpus using the Word2vec software. Third, the dimensional (feature) reduction of BoWs by the intersection between features of BoWs and words in WE to produced NDTMD dataset. Furthermore, the baseline model used BoWs. The third step was the development of classification models used SVM, Logistic regression, and CNN. The fourth step was comparing the performance of the data preprocessing methods used three classification criterion based on feature reduction rate, accuracy, kappa, and running time. The last step 
was to summarize the best approach for short text classification. The experimental framework is shown in Fig. 3.

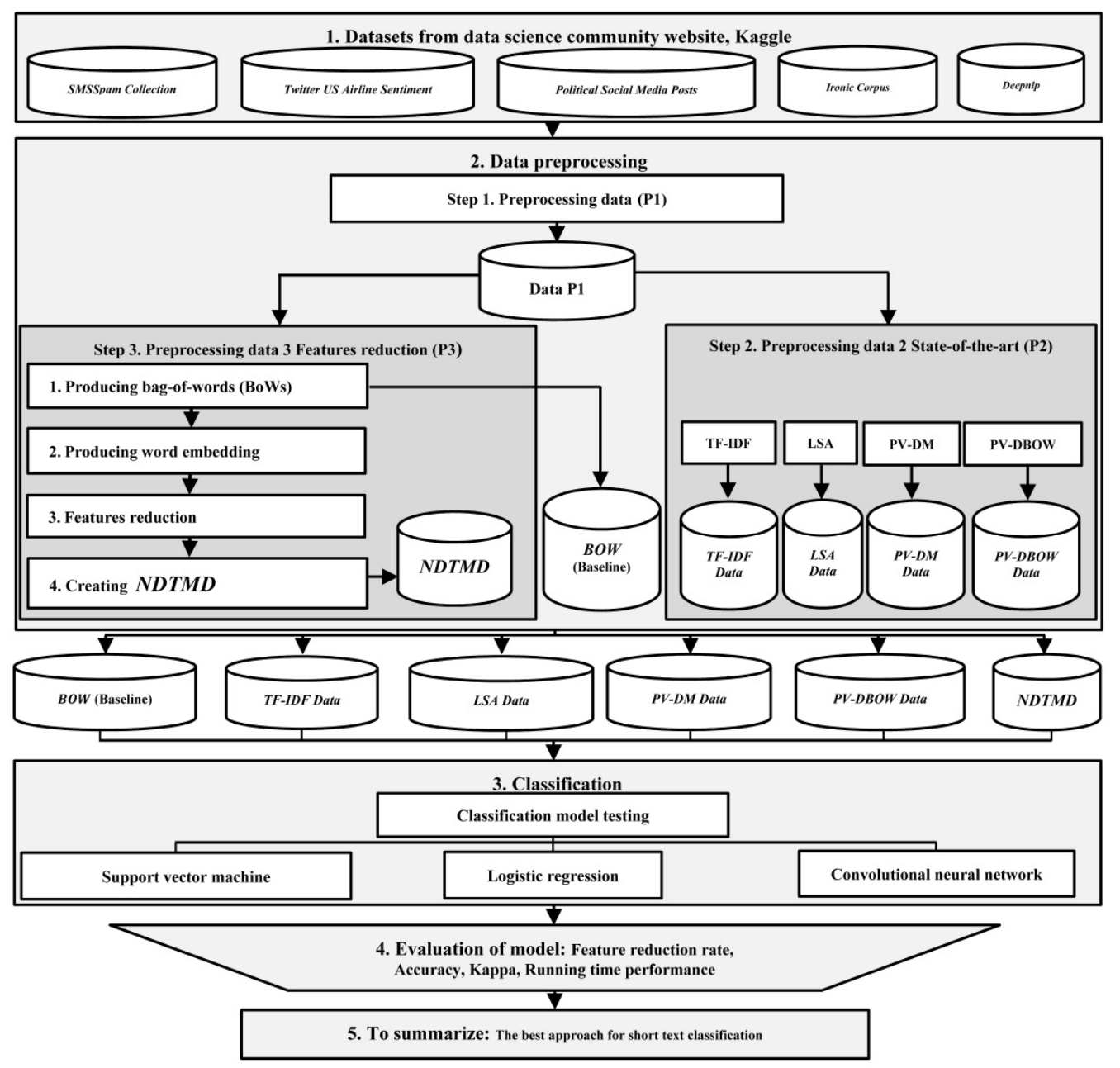

Fig. 3 Experimental framework

\subsection{DATASETS}

In this experiment, the authors selected 5 datasets from the data science community website, Kaggle; [17]. In particular, the authors chose five short text datasets as the input of this experiment.

1) SMSSpamCollection is the collection of SMS messages tagged as spam or ham (not spam). It contains one set of 5,574 SMS messages in English, tagged according to being ham or spam. This dataset has numbers of feature as 50,277 [17]. 2) Twitter US Airline Sentiment is Crowd flower's Data for everyone library. This dataset is the analysis of public reception of each major US airline. Twitter data were sourced from February 2015 and the contributors were asked to classify their tweets as positive, negative or neutral. This dataset has 162,123 features. Furthermore, this dataset is open dataset which is free for everyone to download [17]-[18]. 3) Political Social Media Posts is Crowd flower's Data for everyone library. This dataset provides text of 5,000 messages from politician's social media accounts that along with human judgments. Furthermore, messages purposed partisanship and audience. This dataset has 97,027 features [17]-[18]. 4) Ironic Corpus contains 1,949 comments, which have been labeled as ironic or not ironic by 
human annotators. The text was taken from comments in Reddit. This dataset has numbers of feature as 40,326 [17]. 5) Deepnlp contains 80 user responses to a therapy chatbot that said describe a time when user has acted as a resource for someone that user responded. If the response is not flagged then the user can continue talking to the bot. If it is flagged then the user is referred to help. This dataset has 60,197 features [17]. The specific characteristics of the datasets are shown in Table 1.

Table I. Characteristics of Datasets

\begin{tabular}{|c|c|c|c|c|c|}
\hline Dataset & Instances & $\begin{array}{c}\text { Number } \\
\text { of Classes }\end{array}$ & $\begin{array}{c}\text { Number } \\
\text { of Class Members }\end{array}$ & $\begin{array}{c}\text { Average Sentence } \\
\text { Length (characters) }\end{array}$ & Features (words) \\
\hline SMSSpam Collection & 5,574 & 2 & $\begin{array}{r}\text { 747 SPAM } \\
4,827 \text { HAM } \\
\end{array}$ & 58 & 50,277 \\
\hline Twitter US Airline Sentiment & 14,640 & 3 & $\begin{array}{r}3,099 \text { neutral } \\
2,363 \text { positive } \\
9,178 \text { negative }\end{array}$ & 115 & 162,123 \\
\hline Political-Social-Media-Posts & 5,000 & 2 & $\begin{array}{r}1,311 \text { partisan } \\
\text { 3,689 neutral } \\
\end{array}$ & 214 & 97,027 \\
\hline Ironic Corpus & 1,949 & 2 & $\begin{array}{r}537 \text { ironic } \\
1,412 \text { non-ironic }\end{array}$ & 241 & 40,326 \\
\hline Deepnlp & 204 & 2 & $\begin{array}{r}58 \text { flagged } \\
146 \text { not_flagged }\end{array}$ & 3,254 & 60,197 \\
\hline
\end{tabular}

\subsection{DATA PREPROCESSING}

The data-preprocessing consisted of three steps: P1 as corpus preparation, P2 as state of the art, and P3 as features reduction of datasets.

3.2.1. P1 (CORPUS PREPARATION): P1 consisted of lowering, removing punctuation, removing numbers, removing tabs, removing blank spaces at the beginning, removing stop word, and stemming words to a common base form also results of P1 were Corpus.

Definitions: Corpus $=$ Set of documents from P1

Corpus $=\left\{\operatorname{doc}_{1}, d_{0} c_{2}, d o c_{3}, \ldots, d o c_{d}\right\}$ where $d$ is the number of all documents.

3.2.2. P2 (STATE-OF-THE-ART): $P 2$ which was modern and using the most recent methods. Furthermore, P2 includes TF-IDF, LSA, PV-DM, and PV-DBOW.

1) TF-IDF is a weight that reflects a word importance. Furthermore, a weight uses to evaluate how important a word in corpus [3]. 2) LSA extracts the contextual meaning of words. LSA is used in statistical computations that are applied to represent the corpus [4]-[5]. 3) PV-DM acts as a memory which remembers missing components from the current context or paragraph's topic [6]. And 4) PV-DBOW disregards context words in input but forces models to predict sampling from input paragraphs. PV-DBOW samples a text window and a random word from the text window given the paragraph vector [6].

\subsubsection{PREPROCESSING DATA 3 (FEATURES REDUCTION OF}

DATASET): P3 consists of the proposed NDTMD and including four steps.

1) Producing bag-of-words (BoWs) used a Vectorizer Function in the $R$ programming package [19] to create BoWs. This BoWs represented features through the one-hot encoding of the index. Moreover, an index was a vector filled with zeroes except for a single 1 at the position associated with this index.

Definitions: $B=$ Set of Words in BoWs

$$
\text { BoWs }=\operatorname{Vectorizer(Corpus)}
$$

Corpus is input dataset of BoWs. 
BoWs $=\left\{\right.$ bow $_{1}$, bow $_{2}$, bow $_{3}, \ldots$, bow $\left._{c}\right\}$ where bow is each bag of words in BoWs and $c$ is the numbers of bow in $B o W s$.

$B=\left\{w_{1}, w_{2}, \ldots, w_{m}\right\}$ where $m$ is the number of words in BoWs.

2) Producing word embedding (WE) used $R$ Programming package [19] for interfacing Word2vec Function and producing WE [9]. The skip-gram model used for computing vector representation of WE. The parameters of the skip-gram model were: Window Size $=5$, Vectors Size $=100$, Minimum Count $=5$, Minimum Reduce $=1$, Iteration $=50$.

Definitions: $E$ is set of the words in $W E$.

$\vec{V}$ is vector of words in $W E$.

WE $=$ Word2vec(Corpus)

Corpus is input that produces the dataset as $W E, E=\left\{w_{1}, w_{2}, \ldots, w_{e}\right\}$ where $w$ is each word in $W E$ and $e$ is the numbers of words in $W E, \vec{V}=\left\{\vec{v}_{1}, \vec{v}_{2}, \ldots, \vec{v}_{100}\right\}$ where each word in $W E$ has 100 dimensionality of the vectors.

3) Features reduction, the authors reduced the dimension of BoWs by also creating a document-term matrix (DTM) which was derived from the intersection between words in WE and words in BoWs. DTM was a vector space model whose structure was similar to BoWs but the number of features in DTM was lower than in BoWs.

Definitions: $T$ is set of words that derive from the intersection between set $E$ and $B$ are shown in Equation (7).

$T=E \cap B$

Definitions: $D T M$ is a vector space model.

$D T M=B o W s$ with only words in $T$

$D T M=\left\{w_{1}, w_{2}, \ldots, w_{x}\right\}$ where $w$ is a set of word from DTM and $x$ is the number of words in DTM. Furthermore, DTM $=\left\{\right.$ bow $_{1}$, bow $_{2}$, bow $_{3}, \ldots$, bow $\left._{y}\right\}$ where bow is each bag of words in DTM and $y$ is the numbers of bow in DTM.

4) Producing new document-term matrix (NDTMD)

Definitions: $N D T M D$ is a vector space model which removes the row of DTM if the sum of each row is equal to 0 .

$N D T M D=\left\{w_{1}, w_{2}, \ldots, w_{g}\right\}$ where $w$ is a set of words from NDTMD and $g$ is the numbers of words in NDTMD.

$N D T M D=\left\{\right.$ bow $_{1}$, bow $_{2}$, bow $_{3}, \ldots$, bow $\left._{z}\right\}$ where bow is each bag of words in NDTMD and $z$ is the numbers of words in NDTMD.

Furthermore, producing NDTMD includes three steps. First is calculating the sum of all word frequency in each row of DTM. Second, if the sum of all word frequency in each row is equal to 0 . Then delete each row of DTM. Third, creating a new dataset called NDTMD. The algorithm for producing NDTMD is shown in Fig. 4.

\subsection{DEVELOPMENT OF CLASSIFICATION MODELS}

3.3.1. A SPLIT TEST: The authors split datasets into a training dataset contained $80 \%$ and a test dataset containing the remaining $20 \%$. A split test is suitable for large datasets and this technique has high performance running time [20]. 
3.3.2. CLASSIFIERS: The classifiers included SVM, Logistic regression and CNN. For the first, SVM was selected because this algorithm has advantages to prevent overfitting and yield higher accuracy [14]. For the second, Logistic regression uses logit calculations for data that are not necessary have normal distribution according to statistical techniques [15]. For the third, CNN algorithm has advantages as it does not require prior feature selection process [16].

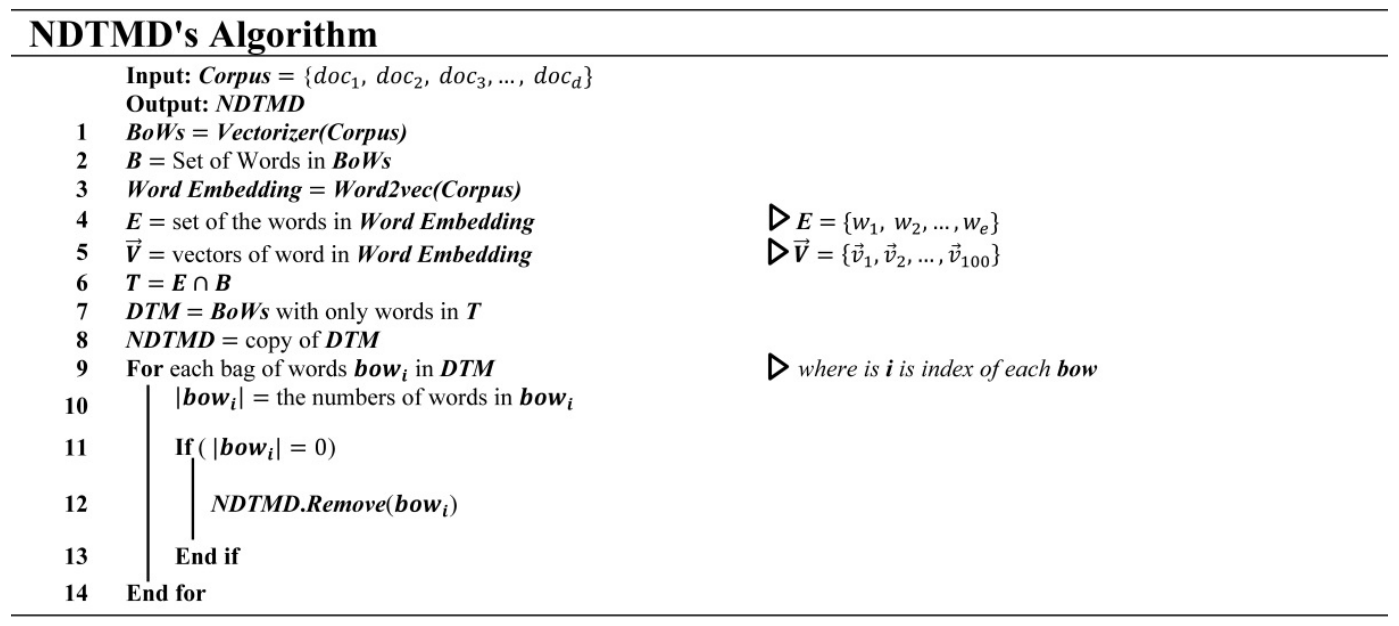

Fig. 4 Algorithm for producing NDTMD

Proof: 1) Let $m$ be the number of words in BoWs and let $e$ be the numbers of words in $W E$ also $e<m$. 2) Let $T$ be the set of words that derives from the intersection of set $E$ and $B$. Thus, the number of words in $T$ must be less than $m$. 3) DTM is derived from $B o W s$ with only words in $T$. Thus, the number of words in DTM must be less than in BoWs. 4) NDTMD is produced by removing (rows) of $D T M$ which the sums of all word frequency are equal to 0 . Let $g$ be the number of words in NDTMD. Therefore, 5) The number of words in NDTMD must be less than in BoWs because of $g<m$.

\subsection{PERFORMANCE MEASUREMENT}

Performance measurements included reduction rate as feature reduction evaluator and the evaluation of the classification performance used the following four performance measures, including FRR, accuracy, kappa and running time.

3.4.1. FEATURE REDUCTION RATE (FRR): The purpose of feature reduction method to choose the most important features while reducing the feature space significantly. This calculation is shown in Equation (8) [21].

$F R R=\frac{(O F-F S)}{O F}$

Where $O F$ is the number of original features. $F S$ is the number of features left after using the feature selection method. Furthermore, FRR is close to 1 which represents high performance to reduce features otherwise FRR is close to 0 which represents low performance.

3.4.2. ACCURACY: Accuracy is the proportion of correct classifications, which is calculated by dividing the number of correctly classified instances by the number 
of total classified instances. Accuracy is widely used to indicate the performance of a classifier. Considers following in Equation (9) [22].

Accuracy $=\frac{T P+T N}{T P+T N+F P+F N}$

3.4.3. KAPPA: Kappa is a metric that indicates the accuracy of a classifier by considering the expected accuracy measured from a random classifier. Kappa can be calculated from the values in a confusion matrix generated in classification modeling. Considers following in Equation (10) [23].

Kappa $=\frac{P_{o}-P_{e}}{1-P_{e}}$

$P_{o}$ is the observed probability of agreement. $P_{e}$ is the hypothetical expected probability of agreement. Kappa value over 0.75 is considered to be excellent while 0.4 to 0.75 is average and under 0.4 is worst [24].

3.4.4. RUNNING TIME: The performance in term of running time of the classification models utilizing the proposed methods is measured and compared. Running time is divided into 3 aspects: Model Time, Train Time, and Test Time. Model Time is the time NDTMD used to select training data. Train Time is the time NDTMD used to classify the whole training set. Test Time is the time NDTMD used to classify the whole test set [25].

\subsection{TO SUMMARIZE}

The authors evaluated the NDTMD method with multiple classification models. The authors compared the performance metrics including FRR, accuracy, kappa and running time. The NDTMD with SVM classifier was the best approach for short text classification model, and the comparison is shown in Section 4.

\section{EXPERIMENTAL RESULTS}

\subsection{EXPERIMENTAL RESULTS OF FEATURES REDUCTION}

The performances of features reduction by NDTMD were compared with the other five preprocessing methods: baseline (BoWs) and four state-of-the-arts consist of TF-IDF, LSA, PV-DM, and PV-DBOW.

Table II. Features Reduction and FRR Performances Using NDTMD

\begin{tabular}{|c|c|c|c|c|c|c|c|c|c|c|c|c|c|}
\hline \multirow[t]{2}{*}{ Dataset } & \multirow{2}{*}{$\begin{array}{c}\text { Features } \\
\text { (words) } \\
\text { of Raw data }\end{array}$} & \multicolumn{6}{|c|}{ Numbers of features } & \multicolumn{6}{|c|}{ Feature Reduction Rate (FRR) } \\
\hline & & $\begin{array}{c}\text { BoWs } \\
\text { (Baseline) }\end{array}$ & TF-IDF & LSA & PV-DM & PV-DBOW & NDTMD & $\begin{array}{c}\text { BoWs } \\
\text { (Baseline) }\end{array}$ & TF-IDF & LSA & PV-DM & PV-DBOW & NDTMD \\
\hline $\begin{array}{l}\text { SMSSpam } \\
\text { Collection }\end{array}$ & 50,277 & 9,757 & 4,340 & 100 & 100 & 100 & 1,513 & 0.8059 & 0.9137 & 0.9980 & 0.9980 & 0.9980 & 0.9699 \\
\hline $\begin{array}{l}\text { Twitter } \\
\text { US Airline } \\
\text { Sentiment } \\
\end{array}$ & 162,123 & 12,815 & 3,234 & 100 & 100 & 100 & 2,572 & 0.9210 & 0.9801 & 0.9994 & 0.9994 & 0.9994 & 0.9841 \\
\hline $\begin{array}{l}\text { Political- } \\
\text { Social } \\
\text {-Media } \\
\text {-Posts } \\
\end{array}$ & 97,027 & 16,384 & 3,650 & 100 & 100 & 100 & 2,724 & 0.8311 & 0.9624 & 0.9990 & 0.9990 & 0.9990 & 0.9719 \\
\hline $\begin{array}{l}\text { Ironic } \\
\text { Corpus }\end{array}$ & 40,326 & 3,919 & 3,658 & 100 & 100 & 100 & 1,386 & 0.9028 & 0.9093 & 0.9975 & 0.9975 & 0.9975 & 0.9656 \\
\hline Deepnlp & 60,197 & 5,804 & 4,245 & 100 & 100 & 100 & 2,278 & 0.9036 & 0.9295 & 0.9983 & 0.9983 & 0.9983 & 0.9622 \\
\hline
\end{tabular}

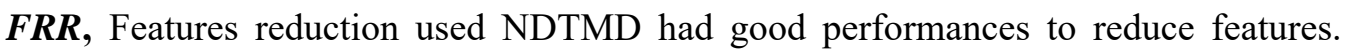
1) NDTMD had a high performance to reduce the number of features of datasets, included SMSSpamCollection (50,277 to 1,513 features), Twitter US Airline Sentiment (162,123 to 2,572 features), Political-Social-Media-Posts (97,027 to 2,724 features), 
Ironic Corpus (40,326 to 1,386 features) and Deepnlp (60,197 to 2,278 features). 2) NDTMD had a high FRR, including SMSSpamCollection (0.9699), Twitter US Airline Sentiment (0.9841), Political-Social-Media-Posts (0.9719), Ironic Corpus (0.9656) and Deepnlp (0.9622). The experimental results of FRR are shown in Table 2. Results show that NDTMD provides the high $(>0.96)$ with all five datasets.

\subsection{EXPERIMENTAL RESULTS OF ACCURACY}

Performance comparisons of classification models were generated by SVM, Logistic regression and $\mathrm{CNN}$ measured by prediction accuracy rates.

Table 3 depicted using $\boldsymbol{S V M}$ classifier. It showed that NDTMD had the highest accuracy rate with three datasets, including Twitter US Airline Sentiment (92.31\%), Ironic Corpus (75.34\%) and Deepnlp (76.92\%).

Table III. Performances of Classification Using SVM and Criterion is Accuracy

\begin{tabular}{|l|r|r|r|r|r|r|}
\hline Dataset & \multicolumn{1}{|c|}{ BOWs (Baseline) } & \multicolumn{1}{|c|}{ TF-IDF } & \multicolumn{1}{c|}{ LSA } & \multicolumn{1}{c|}{ PV-DM } & PV-DBOW & \multicolumn{1}{c|}{ NDTMD } \\
\hline SMSSpamCollection & $\underline{\mathbf{9 8 . 0 3}}$ & 96.86 & 95.87 & 86.62 & 95.87 & 97.78 \\
\hline Twitter US Airline Sentiment & 91.93 & 90.19 & 78.50 & 62.71 & 73.62 & $\underline{\mathbf{9 2 . 3 1}}$ \\
\hline Political-Social-Media-Posts & 74.27 & 73.37 & 76.18 & 73.77 & $\underline{\mathbf{7 6 . 4 8}}$ & 73.97 \\
\hline Ironic Corpus & 73.85 & 70.77 & 73.85 & 73.85 & 73.85 & $\underline{\mathbf{7 5 . 3 4}}$ \\
\hline Deepnlp & 73.17 & 65.87 & 65.37 & 65.37 & 68.29 & $\underline{\mathbf{7 6 . 9 2}}$ \\
\hline
\end{tabular}

Logistic regression, the experimental results showed that NDTMD had high accuracy rate (89.71\%) with Twitter US Airline Sentiment as shown in Table 4.

Table IV. Performances of Classification Using Logistic Regression and Criterion is Accuracy

\begin{tabular}{|l|r|r|r|r|r|r|}
\hline Dataset & \multicolumn{1}{|c|}{ BOWs (Baseline) } & \multicolumn{1}{|c|}{ TF-IDF } & \multicolumn{1}{c|}{ LSA } & \multicolumn{1}{c|}{ PV-DM } & \multicolumn{1}{c|}{ PV-DBOW } & NDTMD \\
\hline SMSSpamCollection & 95.78 & 89.50 & 95.78 & 86.62 & $\underline{\mathbf{9 6 . 9 5}}$ & 96.31 \\
\hline Twitter US Airline Sentiment & $\mathbf{8 9 . 8 6}$ & 89.07 & 75.83 & 62.68 & 71.49 & 89.71 \\
\hline Political-Social-Media-Posts & 73.77 & 66.80 & 76.90 & 73.77 & $\underline{\mathbf{7 6 . 9 0}}$ & 65.57 \\
\hline Ironic Corpus & 53.08 & 60.51 & 71.03 & $\underline{\mathbf{7 2 . 3 4}}$ & 71.79 & 53.89 \\
\hline Deepnlp & $\mathbf{7 0 . 7 3}$ & 60.98 & 58.54 & 53.66 & 63.41 & 66.67 \\
\hline
\end{tabular}

CNN, results showed NDTMD had the highest accuracy with three datasets including Twitter US Airline Sentiment (84.00\%), Ironic Corpus (72.11\%) and Deepnlp (73.17\%) as shown in Table 5.

Table V. Performances of Classification Using CNN and Criterion is Accuracy

\begin{tabular}{|l|r|r|r|r|r|r|}
\hline Dataset & \multicolumn{1}{|c|}{ BOWs (Baseline) } & \multicolumn{1}{|c|}{ TF-IDF } & \multicolumn{1}{c|}{ LSA } & \multicolumn{1}{c|}{ PV-DM } & PV-DBOW & NDTMD \\
\hline SMSSpamCollection & $\mathbf{9 7 . 4 0}$ & 92.74 & 93.54 & 79.10 & 93.72 & 95.93 \\
\hline Twitter US Airline Sentiment & $\mathbf{7 8 . 3 5}$ & 79.92 & 72.71 & 62.12 & 74.18 & $\mathbf{8 4 . 0 0}$ \\
\hline Political-Social-Media-Posts & $\mathbf{7 2 . 5 0}$ & 67.10 & 71.60 & 67.60 & 70.10 & 69.37 \\
\hline Ironic Corpus & 66.41 & 70.25 & 69.49 & 66.41 & 62.31 & $\mathbf{7 2 . 1 1}$ \\
\hline Deepnlp & 60.97 & 56.10 & 68.29 & 63.41 & 70.73 & $\underline{\mathbf{7 3 . 1 7}}$ \\
\hline
\end{tabular}

The results show NDTMD had higher accuracy than other preprocessors by using Twitter US Airline Sentiment dataset when applying three classifiers.

\subsection{EXPERIMENTAL RESULTS OF KAPPA}

SVM results showed that NDTMD had the highest kappa with two datasets including Twitter US Airline Sentiment (0.8559) which was good value, Deepnlp (0.4455). However, BoWs showed the highest kappa (0.9096) for SMSSpamCollection. The experimental results of kappa measures are shown in Table 6. 
Table VI. Classification Performances Using SVM and Kappa

\begin{tabular}{|c|c|c|c|c|c|c|}
\hline Dataset & BoWs (Baseline) & TF-IDF & LSA & PV-DM & PV-DBOW & NDTMD \\
\hline SMSSpamCollection & 0.9096 & 0.8678 & 0.8133 & 0 & 0.7977 & 0.9016 \\
\hline Twitter US Airline Sentiment & 0.8490 & 0.8149 & 0.5668 & 0 & 0.4497 & 0.8559 \\
\hline Political-Social-Media-Posts & 0.3227 & 0.2874 & 0.1835 & 0 & 0.2155 & 0.3087 \\
\hline Ironic Corpus & 0 & 0.0357 & 0 & 0 & 0 & 0 \\
\hline Deepnlp & 0.3218 & 0.1847 & 0 & 0.0831 & 0.1305 & 0.4455 \\
\hline
\end{tabular}

Logistic regression results showed NDTMD had the highest kappa in two datasets including Ironic Corpus (0.0234) and Deepnlp (0.3407) which both kappas are not good value. However, PV-DBOW and BoWs showed the highest and good kappa value in SMSSpamCollection (0.8644) and Twitter US Airline Sentiment (0.8073), respectively. The experimental results of kappa measures are shown in Table 7.

Table VII. Classification Performances Using Logistic Regression and Kappa

\begin{tabular}{|c|c|c|c|c|c|c|}
\hline Dataset & BoWs (Baseline) & TF-IDF & LSA & PV-DM & PV-DBOW & NDTMD \\
\hline SMSSpamCollection & 0.8164 & 0.6315 & 0.8142 & 0 & $\underline{0.8644}$ & 0.8370 \\
\hline Twitter US Airline Sentiment & 0.8073 & 0.7906 & 0.4850 & 0 & $\overline{0.3837}$ & 0.8045 \\
\hline Political-Social-Media-Posts & 0 & 0.1837 & 0.2857 & 0.0549 & 0.2878 & 0.1713 \\
\hline Ironic Corpus & 0.0030 & 0.0718 & 0.0402 & 0.0211 & 0.0149 & 0.0234 \\
\hline Deepnlp & 0.3260 & 0.1323 & 0.0619 & 0.0512 & 0.0149 & $\underline{0.3407}$ \\
\hline
\end{tabular}

CNN results showed NDTMD had the highest kappa with three datasets including Twitter US Airline Sentiment (0.7063) which was good kappa, Ironic Corpus (0.1212) and Deepnlp (0.3930). However, BoWs showed the highest and good kappa value in SMSSpamCollection (0.8971) and Political-Social-Media-Posts (0.2754), respectively. The experimental results of kappa measures are shown in Table 8.

Table VIII. Classification Performances Using CNN and Kappa

\begin{tabular}{|l|r|r|r|r|r|r|}
\hline Dataset & BoWs (Baseline) & \multicolumn{1}{|c|}{ TF-IDF } & \multicolumn{1}{c|}{ LSA } & PV-DM & PV-DBOW & NDTMD \\
\hline SMSSpamCollection & $\underline{\mathbf{0 . 8 9 7 1}}$ & 0.7459 & 0.7515 & 0.0322 & 0.7675 & 0.8249 \\
\hline Twitter US Airline Sentiment & 0.6010 & 0.6237 & 0.4978 & 0.0030 & 0.3979 & $\underline{\mathbf{0 . 7 0 6 3}}$ \\
\hline Political-Social-Media-Posts & $\mathbf{0 . 2 7 5 4}$ & 0.1806 & 0.2176 & 0.1148 & 0.2079 & 0.2486 \\
\hline Ironic Corpus & 0.1164 & 0.0782 & 0.1116 & 0.0066 & 0.0021 & $\underline{\mathbf{0 . 1 2 1 2}}$ \\
\hline Deepnlp & 0.1014 & 0.0238 & 0.2566 & 0.0376 & 0.2454 & $\underline{\mathbf{0 . 3 9 3 0}}$ \\
\hline
\end{tabular}

Thus NDTMD is seemed to be fitted with Twitter US Airline Sentiment dataset which using two classifiers, SVM and CNN to generate classification model since it provided the biggest kappa value.

\subsection{EXPERIMENTAL RESULTS OF RUNNING TIME}

Using $\boldsymbol{S V M}$, the experimental results showed NDTMD had the lowest running time for classification with Ironic Corpus (0.06 seconds). The experimental results are shown in Table 9.

Table IX. Classification Performances by Running Time (Seconds) Using SVM

\begin{tabular}{|l|r|r|r|r|r|r|}
\hline Dataset & \multicolumn{1}{|c|}{ BoWs (Baseline) } & \multicolumn{1}{c|}{ TF-IDF } & \multicolumn{1}{c|}{ LSA } & \multicolumn{1}{c|}{ PV-DM } & \multicolumn{1}{c|}{ PV-DBOW } & \multicolumn{1}{c|}{ NDTMD } \\
\hline SMSSpamCollection & 30.83 & 8.71 & $\mathbf{0 . 0 1}$ & $\mathbf{0 . 0 1}$ & $\mathbf{0 . 0 1}$ & 0.56 \\
\hline Twitter US Airline Sentiment & 1765.70 & 8.71 & 2.50 & 3.34 & $\mathbf{1 . 8 5}$ & 3.23 \\
\hline Political-Social-Media-Posts & 250.50 & 9.89 & $\underline{\mathbf{5 . 8 0}}$ & 6.66 & 6.97 & 10.64 \\
\hline Ironic Corpus & 0.22 & 0.10 & 0.23 & 0.17 & 0.22 & $\mathbf{0 . 0 6}$ \\
\hline Deepnlp & 0.12 & 0.11 & 0.04 & $\mathbf{0 . 0 2}$ & 0.03 & 0.28 \\
\hline
\end{tabular}

When the classifier was Logistic regression the experimental results showed PVDBOW had the lowest running time with three datasets Ironic (Political-SocialMedia-Posts, 0.52 seconds, Ironic Corpus, 0.01 seconds and Deepnlp, 0.03 
seconds). Last, PV-DM had the lowest running time with two datasets: SMSSpamCollection (0.65) and Twitter US Airline Sentiment (0.42). It is likely that Logistic regression does not fit with NDTMD. The experimental results are shown in Table 10.

Table X. Classification Performances by Running Time (Seconds) Using Logistic Regression

\begin{tabular}{|c|c|c|c|c|c|c|}
\hline Dataset & BoWs (Baseline) & TF-IDF & LSA & PV-DM & PV-DBOW & NDTMD \\
\hline SMSSpamCollection & 17.63 & 17.07 & 1.12 & 0.65 & 0.99 & 2.45 \\
\hline Twitter US Airline Sentiment & 893.64 & 8.05 & 1.23 & $\underline{0.42}$ & 2.89 & 4.95 \\
\hline Political-Social-Media-Posts & 2521.57 & 4.13 & 0.91 & $\overline{0.59}$ & $\underline{0.52}$ & 7.69 \\
\hline Ironic Corpus & 0.16 & 0.02 & 0.02 & 0.01 & $\underline{0.01}$ & 0.06 \\
\hline Deepnlp & 557.18 & 47.92 & 0.06 & 0.09 & 0.03 & 33.82 \\
\hline
\end{tabular}

When the classifier was $C N N$ the experimental results show LSA had the lowest running time with three datasets (Twitter US Airline Sentiment, 93.42 seconds, PoliticalSocial-Media-Posts 34.17 seconds, and Deepnlp, 1.22 seconds). NDTMD did not provide the best running time for all datasets. The experimental results are shown in Table 11.

Table XI. Classification Performances by Running Time (Seconds) Using CNN

\begin{tabular}{|l|r|r|r|r|r|r|}
\hline Dataset & \multicolumn{1}{|c|}{ BoWs (Baseline) } & \multicolumn{1}{|c|}{ TF-IDF } & \multicolumn{1}{c|}{ LSA } & \multicolumn{1}{c|}{ PV-DM } & \multicolumn{1}{c|}{ PV-DBOW } & NDTMD \\
\hline SMSSpamCollection & 94.00 & 64.33 & 40.59 & $\underline{\mathbf{9 9 . 2 3}}$ & 35.08 & 35.69 \\
\hline Twitter USAirline Sentiment & 1644.40 & 1211.27 & $\underline{\mathbf{9 3 . 4 2}}$ & 94.15 & 98.36 & 146.02 \\
\hline Political-Social-Media-Posts & 786.17 & 125.34 & $\underline{\mathbf{3 4 . 1 7}}$ & 34.29 & 34.50 & 47.55 \\
\hline Ironic Corpus & 19.77 & 24.36 & 15.27 & 10.99 & $\underline{\mathbf{1 0 . 0 5}}$ & 14.64 \\
\hline Deepnlp & 10.40 & 8.24 & $\underline{\mathbf{1 . 2 2}}$ & 1.22 & 1.36 & 1.85 \\
\hline
\end{tabular}

It is likely that NDTMD worked well with SVM classifier since it provided the smallest running time in classification of two datasets: SMSSpamCollection and Ironic Corpus.

\section{DISCUSSION}

Based on Mikolov's suggestion, the skip-gram model (which was used to produce NDTMD), provides good results for infrequent words. Thus, the feature reduction of short text classification produced NDTMD by the intersection between features of BoWs and words in WE which were generated from the corpus using the Word2vec software, the resulting NDTMD was effective at handling infrequent words in the dataset, such as technical terms or nomenclature. Moreover, this implementation is different to BoWs as the latter reduces features by removing infrequent words. An advantage of the skip-gram model is that it produces a better vector when applied to infrequent words because Mikolov modified the optimization objective that resulting in a small number of updates to the weight of model with a technique called "Negative Sampling" [8].

The proposed the feature reduction of short text classification by NDTMD used feature intersection of BoWs and words in WE. In contrast, it was different to the former papers by Lilleberg et al. [12] and Kenter and Rijki [13]. Since, NDTMD reduced the size of BoWs features. While, Lilleberg et al. [12] combined the features of Word2vec and TF-IDF. Moreover, Kenter and Rijki [13] concatenated features of WE and words in sentences using for short text classification. Furthermore, NDTMD had a high FRR which represents high performance to reduce features as well. NDTMD provided the highest FRR rate of 0.9841 in Twitter US Airline Sentiment dataset. When the NDTMD was used in the classification, the input data of NDTMD were smaller than BoWs and TF-IDF, making NDTMD more efficient. Moreover, the new method provided the higher 
accuracy than BoWs, TF-IDF, LSA, PV-DM and PV-DBOW. The CNN classifier using NDTMD had accuracy rate of $84.00 \%$ (Twitter US Airline Sentiment), $72.11 \%$ (Ironic Corpus) and 73.17\% (Deepnlp). Furthermore, this new method provided the highest accuracy rate of $92.31 \%$ (Twitter US Airline Sentiment), $75.34 \%$ (Ironic Corpus) and 76.92\% (Deepnlp) with SVM classifier.

\section{CONCLUSION}

The authors proposed a new method NDTMD which is the features reduction of BoWs. This article solved the weaknesses of BoWs which is a high dimensional vector. The experiment included four steps. In the first step, 5 datasets were undertaken from the data science community website, Kaggle. The second step was included data preprocessing that included three steps. P1 was corpus preparation. P2 were various states of the art data preprocessing. P3 was new data preprocessing methods for features reduction of the dataset using WE. First, BoWs was generated from corpus and WE was generated from corpus by using the Word2 $\mathrm{vec}$ software. Next, the features reduction of BoWs performed by the intersection between features of BoWs and words in WE that produced NDTMD data, the baseline model used BoWs. The third step was the development of classification models used SVM, Logistic regression, and CNN. The fourth step was evaluating the performance of data preprocessing methods using three algorithms (SVM, Logistic regression, $\mathrm{CNN}$ ) for text classification. Moreover, the authors compared the classification criterion by using accuracy, kappa, and running time. Then, the authors compared the performances of FRR. NDTMD methods had the highest FRR as well. NDTMD had higher accuracy than other preprocessors when using Twitter US Airline Sentiment dataset and applying three classifiers. For kappa value, NDTMD is seemed to be fitted with Twitter US Airline Sentiment dataset which using two classifiers, SVM and CNN to generate classification model since it provided the biggest kappa value. Last, NDTMD worked well with SVM classifier since it provided the smallest running time in classification of Ironic Corpus datasets. It is likely that NDTMD worked well and most fitted with Twitter US Airline Sentiment dataset which is short (with average 115 characters/sentences). Thus, the best approach for short text classification model was using NDTMD with SVM classifier.

Future work could consider the interesting results in a short text dataset having high numbers of attributes, such as in the datasets of Twitter US Airline Sentiment, Ironic Corpus and Deepnlp. Twitter US Airline Sentiment, Ironic Corpus and Deepnlp had the highest accuracy when the classification model used SVM. Furthermore, it had high kappa with SVM model. Thus, for the future, the authors are interested in applying the proposed techniques to real data on social media.

\section{ACKNOWLEDGMENTS}

The authors thank the Department of Computer Science, Faculty of Science, Kasetsart University, Bangkok, Thailand for financial support. The authors thank Dr. Chia-Hui Chang, Professor at the National Central University, Taiwan for providing much useful comments.

\section{REFERENCES}

[1] Harris, Zellig S. "Distributional Structure”, Word, vol. 10, no. 2-3, (1954): 146-162.

[2] Jörg A., Walter and Helge, Ritter. "On Interactive Visualization of High-Dimensional Data Using the Hyperbolic Plane", International Conference on Knowledge Discovery and Data Mining. The eighth ACM SIGKDD, 2002.

[3] Leskovec, Jure, et al. Mining of Massive Datasets. Cambridge university press, 2014. 
[4] Dumais, Susan T. "Latent Semantic Analysis", Annual Review of Information Science and Technology. 38, (2005): 188-230.

[5] Landauer, Thomas K., et al. "An Introduction to Latent Semantic Analysis", Discourse Processes, vol. 25, no. 2-3, (1998): 259-284.

[6] Quoc, Le and Tomas, Mikolov. "Distributed Representations of Sentences and Documents", International Conference on Machine Learning, 2014, pp. 1188-1196.

[7] Mikolov, Tomas, et al. "Efficient Estimation of Word Representations in Vector Space", ICLR Workshop, (2013).

[8] Mikolov, Tomas, et al. "Distributed Representations of Words and Phrases and Their Compositionality", Advances in Neural Information Processing Systems, (2013): 3111-3119.

[9] Google Teams. "Word2vec-Google Code Archive-Long-Term Storage for Google Code Project Hosting", https://code.google.com/archive/p/word2vec

[10] Peng, Jin, et al. "Bag-of-Embeddings for Text Classification", Twenty-Fifth International Joint Conference on Artificial Intelligence (IJCAI-16), 2016, pp. 2824-2830.

[11] Michael, Gutmann and Aapo, Hyvärinen. "Noise-Contrastive Estimation: A New Estimation Principle for Unnormalized Statistical Models", The Thirteenth International Conference on Artificial Intelligence and Statistics, 2010, pp. 297-304.

[12] Joseph, Lilleberg, et al. "Support Vector Machines and Word2Vec for Text Classification with Semantic Features", Cognitive Informatics \& Cognitive Computing, 2015 IEEE 14th International Conference on, 2015, pp. 136-140.

[13] Tom, Kenter and Maarten, de, Rijke. "Short Text Similarity with Word Embeddings", The 24th ACM International on Conference on Information and Knowledge Management, 2015, pp. 1411-1420.

[14] Cortes, Corinna and Vapnik, Vladimir. "Support-Vector Networks", Machine learning, vol. 20, no. 3, (1995): 273-297.

[15] Walker, Strother H. and Duncan, David B. "Estimation of the Probability of an Event as a Function of Several Independent Variables”, Biometrika, vol. 54, no. 1-2, (1967): 167-179.

[16] Kim, Yoon. "Convolutional Neural Networks for Sentence Classification”, arXiv preprint arXiv:1408.5882, (2014).

[17] Kaggle Inc. "Terms and Conditions - Kaggle”, https://www.kaggle.com/datasets

[18] Figure Eight Inc. "Data for Everyone”, https://www.figure-eight.com/data-for-everyone/

[19] R Core Teams. "R: A Language and Environment for Statistical Computing", R Foundation for Statistical Computing, Vienna, Austria. ISBN 3-900051-07-0, URL http://www.R-project.org/

[20] Witten, Ian H., et al. Data Mining: Practical Machine Learning Tools and Techniques. Morgan Kaufmann, 2016.

[21] Hsin-Yu, Ha, et al. "FC-MST: Feature Correlation Maximum Spanning Tree for Multimedia Concept Classification", Computing (ICSC), IEEE International Conference, 2015, pp. 276-283.

[22] Xiangfeng, Dai and Robert, Prout. "Unlocking Super Bowl Insights: Weighted Word Embedding for Twitter Sentiment Classification", The 3rd Multidisciplinary International Social Networks Conference on SocialInformatics, 2016, pp. 12.

[23] Viera, Anthony J. and Garrett, Joanne M. "Understanding Interobserver Agreement: The Kappa Statistic", Fam Med, vol. 37, no. 5, (2005): 360-363.

[24] Davis, Adrian T. and Moed, Berton R. "Can Experts in Acetabular Fracture Care Determine Hip Stability After Posterior Wall Fractures Using Plain Radiographs and Computed Tomography”, Journal of Orthopaedic Trauma, vol. 27, no. 10, (2013): 587-591.

[25] Edouard, Grave, et al. "Bag of Tricks for Efficient Text Classification", The 15th Conference of the European Chapter of the Association for Computational Linguistics, EACL, 2017, pp. 3-7. 
International Journal of Control and Automation Vol. 12, No. 2, (2019) 